\section{Haemophilus influenza b : la lutte avance...}

Caroline Charlier-Woerther, Olivier Lortholary
Centre d'Infectiologie Necker-Pasteur,

Hôpital Necker-Enfants malades,

149 , rue de Sèvres,

75743 Paris Cedex 15, France.

Institut Pasteur, 25, rue Docteur Roux, 75015

Paris, France.

caro_charlier@yahoo.fr vaccination supplémentaire [2, 3].

Parmi les questions en suspens persistent la nécessité d'assurer une couverture vaccinale satisfaisante dans tous les districts, celle d'effectuer une surveillance correcte et complète des cas de Hib au même titre que des autres agents responsables de pneumopathies ou de méningites, ce qui n'est pas encore le cas actuellement.

Cependant, ces résultats majeurs montrent comment, à l'échelle continentale, volonté politique forte et mise en œuvre réelle de moyens satisfaisants permettent la réduction majeure de la morbimortalité associée à un pathogène; ils font de cette stratégie un modèle exportable pour d'autres politiques d'introduction vaccinale, dans d'autres régions du globe. $\diamond$

Haemophilus influenza $b$ :

on the good track

\section{RÉFÉRENCES}

1. Danovaro-Holliday MC, Garcia S, de Quadros C, et al. Progress in vaccination against Haemophilus influenzae type b in the Americas. PLos Med 2008; 5 : e87.

2. Hanslik T, Boëlle Py. L'évaluation du rapport risque/ bénéfice des stratégies de vaccination. Med Sci (Paris) $2007 ; 23: 391-8$

3. Duclos P, Okwo-Bele JM. Recommandations et politiques vaccinales mondiales : le rôle de l'OMS. Med Sci (Paris) 2007 ; $23: 409-16$. l'importance de la morbi-mortalité liée à Hib et l'impact favorable de politiques vaccinales, la diffusion de ces données régional de I'OMS qui facilite notam-
ment l'achat des vaccins et recueille
$>$ Avec à son actif un bilan de 400000 déces annuels par meningite ou type $b$ (Hib) figure en bonne place parmi les pathogènes «tueurs d'enfants » comme les agents de la tuberculose ou du paludisme. Il est aussi responsable d'une morbidité infantile majeure sous la forme d'épiglottites, de septicémies, d'arthrites et surtout de séquelles neurologiques sévères affectant jusqu'à $30 \%$ des enfants survivant à une méningite. cinale systématique des enfants s'est appuyée sur l'aide du PAHO (pan american health organization), bureau les données de surveillance (incidence, et de l'expérience acquise parmi les pays concernés. Ils insistent également sur les efforts mis en jeu par la PAHO pour assurer la viabilité financière du projet au moment d'introduire le surcoût d'une En 2006, tous les pays américains en les efforts (n) 